\title{
DBR and DFB lasers in neodymium- and ytterbium-doped photothermorefractive glasses
}

\author{
A. Ryasnyanskiy, ${ }^{1, *}$ N. Vorobiev, ${ }^{2}$ V. Smirnov, ${ }^{1}$ J. Lumeau ${ }^{2,3}$ L. Glebova, ${ }^{1}$ O. Mokhun, ${ }^{1}$ Ch. Spiegelberg, ${ }^{1}$ \\ Michael Krainak, ${ }^{4}$ A. Glebov, ${ }^{1}$ and L. Glebov ${ }^{2}$ \\ ${ }^{1}$ OptiGrate Corp, 562 South Econ Circle, Oviedo, Florida 32765, USA \\ ${ }^{2}$ CREOL-The College of Optics and Photonics, University of Central Florida, Orlando, Florida 32816-2700, USA \\ ${ }^{3}$ Aix-Marseille Université, CNRS, Centrale Marseille, Institut Fresnel, UMR 7249, Marseille 13013, France \\ ${ }^{4}$ NASA Goddard Space Flight Center, 8800 Greenbelt Rd., Greenbelt, Maryland 20771, USA \\ ${ }^{*}$ Corresponding author: aryasnyanskiy@optigrate.com
}

Received January 3, 2014; revised March 5, 2014; accepted March 10, 2014; posted March 11, 2014 (Doc. ID 204149); published March 31, 2014

\begin{abstract}
The first demonstration, to the best of our knowledge, of distributed Bragg reflector (DBR) and monolithic distributed feedback (DFB) lasers in photothermorefractive glass doped with rare-earth ions is reported. The lasers were produced by incorporation of the volume Bragg gratings into the laser gain elements. A monolithic single-frequency solid-state laser with a linewidth of $250 \mathrm{kHz}$ and output power of $150 \mathrm{~mW}$ at $1066 \mathrm{~nm}$ is demonstrated. (C) 2014 Optical Society of America

OCIS codes: (140.3490) Lasers, distributed-feedback; (140.3580) Lasers, solid-state; (160.5690) Rare-earth-doped materials; (050.7330) Volume gratings.

http://dx.doi.org/10.1364/OL.39.002156
\end{abstract}

The need for environment-insensitive, compact, robust, narrow line laser sources has stimulated the development of hybrid devices, such as distributed Bragg reflector (DBR) lasers, in which a laser resonator is produced by Bragg mirrors incorporated in a gain element, or distributed feedback (DFB) lasers, in which a resonator is produced by a Bragg grating that occupies the whole gain element. The concept of DFB was first successfully applied to optically pumped dye lasers [1,2], in which the feedback was provided by a spatial periodic refractive index (or gain) variation in dye-doped thin films. DFB or DBR grating structures have been used to demonstrate lasing in various types of organic/polymer thin films and waveguide structures since then. The first observation of a successful DFB laser in GaAs semiconductor laser was reported in 1973 [3]. Since then, semiconductor DFB lasers have seen a huge technological development as these structures are compact, provide a high degree of spectral selection, and do not require polished end reflectors to facilitate coupling to other monolithic circuit components. The standard approach for the realization of single-mode edge-emitting lasers is the incorporation of a grating in the laser resonator, either in a DFB or a DBR structure. The grating period and the effective refractive index of the laser waveguide determine the emission wavelength of the laser. Output power of several hundred milliwatts has been realized with this approach [ $\underline{4}-\underline{6}]$.

Recent development of photothermorefractive (PTR) glass for recording volume holograms has led to the creation of high-efficiency low-loss volume Bragg gratings (VBGs) $[\underline{7}, \underline{8}$. The main advantage of such elements is that they allow for narrowband filters in both spectral and angular spaces and are suitable for high power laser applications [9]. It was found that codoping of PTR glass with such a luminescent ion as Nd results in a complex material that combines both photosensitivity and highefficiency luminescence [10]. Lasing of diode-pumped Nd-doped PTR glass in an external resonator was demonstrated [11]. It is clear that recording VBGs inside the laser glass should pave the way for creation of monolithic DFB and DBR solid-state lasers. Such lasers should enable a dramatic increase of aperture and provide dramatic increases in the power of single frequency lasers in comparison with fiber, diode, or thin film organic DFB lasers. Thus, the goal of the current work is to create and investigate DFB or DBR lasers in PTR glass doped with rare-earth ions.

The PTR glass has composition (M\%) $14 \mathrm{Na}_{2} \mathrm{O}-4 \mathrm{NaF}-$ $1 \mathrm{KBr}-5 \mathrm{ZnO}-2 \mathrm{Al}_{2} \mathrm{O}_{3}-1 \mathrm{AlF}_{3}-72 \mathrm{SiO}_{2}-0.01 \mathrm{Ag}_{2} \mathrm{O}-0.01 \mathrm{Ce}_{2} \mathrm{O}_{3}-0.01$ $\mathrm{SnO}_{2}-0.01 \mathrm{Sb}_{2} \mathrm{O}_{3}[\underline{7}, 8]$. The two last elements are responsible for initiation of the photostructural transformations in the glass and enable VBG recording. As it was demonstrated in our earlier studies [10], additional doping with rare-earth ions allows fabricating gain elements for lasers with external resonators produced by conventional and Bragg mirrors. The main concern of incorporating resonator mirrors inside of PTR glass gain elements was the unpredictable competing interaction between different rare-earth components that provide different functionality, which are a photosensitizer $\mathrm{Ce}$ and luminescent agents $\mathrm{Nd}$ or $\mathrm{Yb}$.

Nd- and Yb-doped PTR glasses with 2 wt. \% of $\mathrm{Yb}$ and Nd ions have been prepared. The measurements of emission spectra were carried out in these glasses using an Ocean Optics spectrometer when glass samples were excited with a diode laser emitting at $808 \mathrm{~nm}$ (in the case of $\mathrm{Nd}$ ions) and $915 \mathrm{~nm}$ (for $\mathrm{Yb}$ ones) (Fig. 1). One can see that this glass presents broadband emission spectra typical for silicate glasses, demonstrating the possibility for wide range tunability. The measurements of luminescence lifetime were performed using photodiodes when glass was excited with modulated diode lasers emitting at 808 and $915 \mathrm{~nm}$. The luminescence decay shape was close to an exponential function. The extracted lifetimes of Nd- and $\mathrm{Yb}$-doped PTR glasses with $2 \mathrm{wt}$ \% of Nd and $\mathrm{Yb}$ ions were found to be $\sim 0.8$ and $\sim 3 \mathrm{~ms}$ respectively. The cross sections of emission in the vicinity of $1060 \mathrm{~nm}$ is $1.2 \times 10^{-20} \mathrm{~cm}^{2}$ for $\mathrm{Nd}$ and $0.8 \times 10^{-21} \mathrm{~cm}^{2}$ 


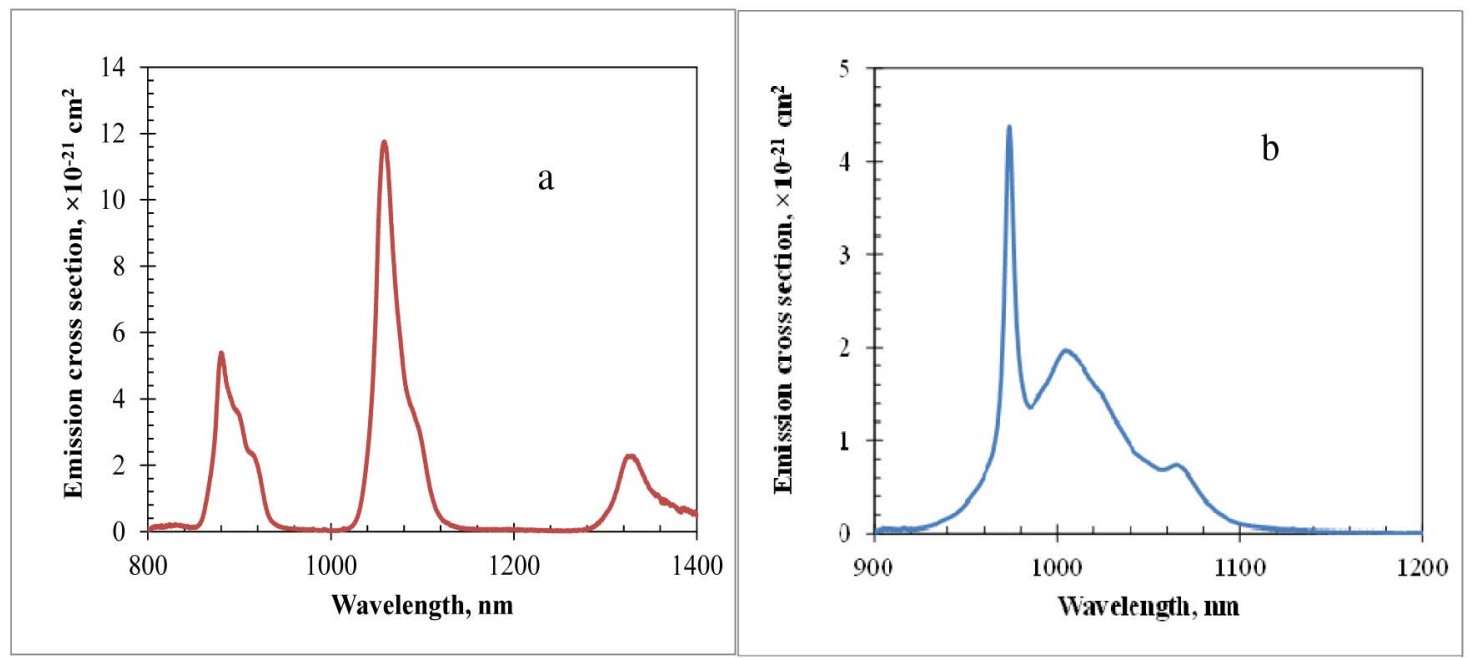

Fig. 1. Emission spectra of PTR glasses with $2 \%$ of $\mathrm{Nd}$ ions (a) and $2 \%$ of $\mathrm{Yb}$ ions (b) under Xe lamp excitation.

for $\mathrm{Yb}$. These numbers are typical for silicate laser glasses.

Photosensitivity (refractive index change) of these glasses was measured by means of a shearing interferometer after exposure of glasses to UV radiation at $325 \mathrm{~nm}$ followed by thermal development at temperatures above $500^{\circ} \mathrm{C}[12]$. It was found that both glasses provide refractive index change up to about $500 \mathrm{ppm}$ that is sufficient for recording VBGs with high efficiency.

Volume holograms were recorded by interferometric patterns. The strength of recorded gratings was evaluated by measuring relative diffraction efficiency (RDE). This allows separating the efficiency of recorded gratings from absorption caused by dopants. RDE was calculated from the intensity of light transmitted through a hologram at the Bragg condition and out of Bragg resonance.

Laser setups for DBR and DFB laser system are presented in Fig. 2.

In the case of a DBR laser [Fig. 2(a)], the cavity consists of a broadband mirror with high transmission at $808 \mathrm{~nm}$ and high reflection in the vicinity of $1060 \mathrm{~nm}$ (1), a $50 \mathrm{~mm}$ focusing lens (2), and a DBR element (3) that acted as both a gain element (Nd- or Yb-doped PTR glass) and an output coupler (a VBG with diffraction efficiency of $\sim 99 \%)$. This telescope-type cavity configuration enables stable operation of the laser.
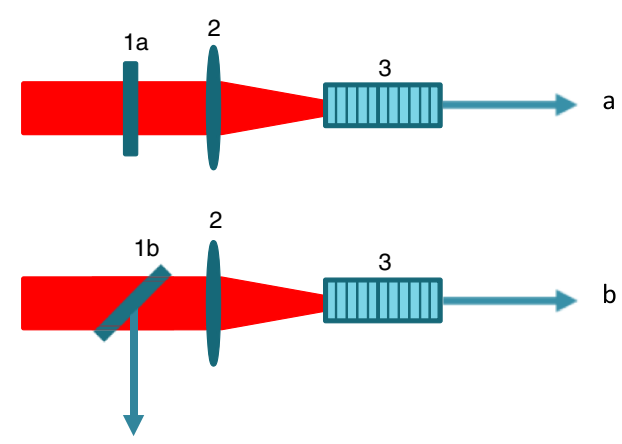

Fig. 2. Experimental setups of DBR (a) and DFB (b) lasers. 1, dichroic mirror with high transmission for pumping radiation and high reflection for signal at normal incidence (a) or at $45^{\circ}$ (b); 2, lens; 3, Nd- or Yb-doped PTR glass slab with a 99\% diffraction efficiency Bragg mirror.
In the case of a DFB laser [Fig. 2(b)], no external elements for a resonator were used. The cavity length was entirely determined by the length of an active element (10-15 mm). Since a DFB laser has two outputs, a dichroic beam splitter (1b) was placed before the focusing lens in order to protect the laser diode from the backpropagating laser beam.

In the case of Nd-doped elements, an $808 \mathrm{~nm}$ pump source with a maximum power of $40 \mathrm{~W}$ was used, whereas in the case of $\mathrm{Yb}$-doped samples, a fiber-coupled $976 \mathrm{~nm}$ laser diode with a maximum power of $30 \mathrm{~W}$ was used. To prevent heating of a Nd-doped active element, the pumping was in a pulsed regime with a pulse duration of $1 \mathrm{~ms}$ and repetition rate of $50 \mathrm{~Hz}$. The DBR and DFB elements with antireflection coating on both facets with an aperture of $2 \mathrm{~mm} \times 6 \mathrm{~mm}$ and a thickness of 10-15 mm were kept in a copper housing mounted on the thermoelectric cooler (TEC) for temperature control.

As an active laser element, we used a PTR glass sample doped with $2 \%$ of Nd ions. The Bragg mirror recorded inside the volume of an active element had a RDE of $99 \%$. Lasing with a pulse width of about $1 \mathrm{~ms}$ was at a threshold of $12 \mathrm{~W}$ of absorbed pump power. The output characteristics of the laser are presented in Fig. 3. The maximum output peak power was measured to be $3 \mathrm{~W}$ (pulse energy, $6 \mathrm{~mJ}$; slope efficiency, 15\%) without any signature of saturation due to thermal lens effects and, therefore, was limited by the pump power level [Fig. 3(a)]. Optical-to-optical conversion efficiency was measured to be $7.5 \%$. Measurements of the spectral line width [Fig. 3(b)] showed a narrow line limited by the resolution of the optical spectrum analyzer ( $\sim 30 \mathrm{pm})$.

It was found that increasing the pump power resulted in a redshift of the emitted central wavelength of the laser. Thus, $\sim 350 \mathrm{pm}$ redshift was observed [Fig. 3(c)] at maximum pump level ( $30 \mathrm{~W}$ of absorbed peak power) in comparison with a threshold $(12 \mathrm{~W}$ of absorbed peak power). This effect can be explained as follows. Increase of the pump power results in a higher rate of heat generation caused by nonradiative relaxation of the Nd ions.

As a consequence, the local temperature within a pumped area in the DBR element increased. Heating 


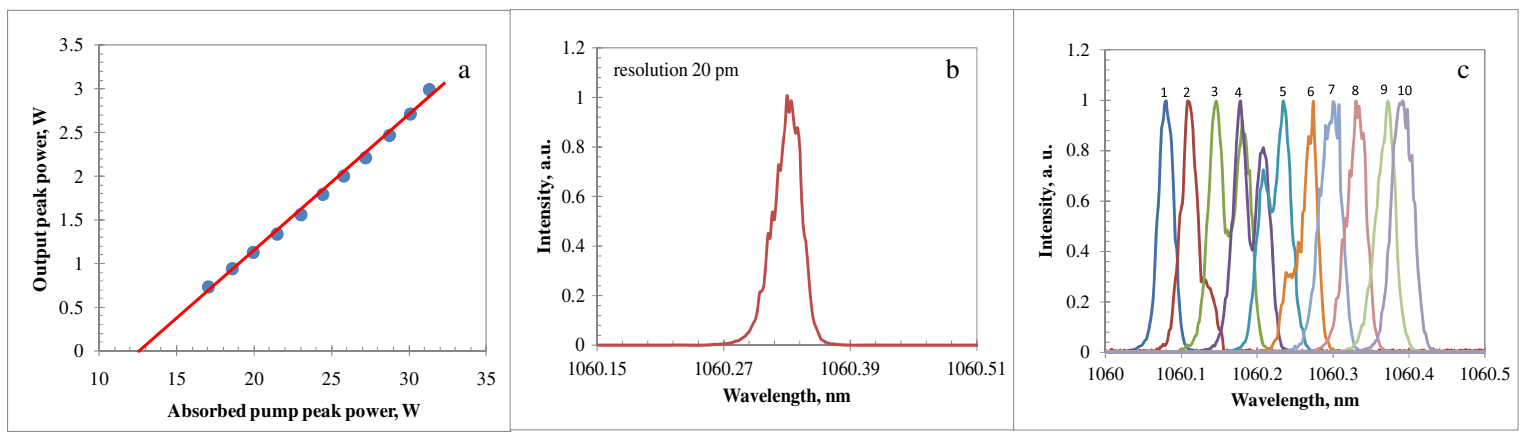

Fig. 3. Output parameters of a Nd:PTR glass DBR laser. (a) shows peak power versus absorbed pump power. (b) Emission spectrum at $28.7 \mathrm{~W}$ of absorbed pump power. (c) Emission spectra at different values of absorbed pump power: 1, 17.1 W; 2, $18.6 \mathrm{~W} ; 3$, $20 \mathrm{~W} ; 4,21.5 \mathrm{~W} ; 5,24.4 \mathrm{~W} ; 6,25.8 \mathrm{~W} ; 7,27.2 \mathrm{~W} ; 8,28.7 \mathrm{~W} ; 9,30 \mathrm{~W} ; 10,31.3 \mathrm{~W}$.

of PTR glass results in thermal expansion with coefficient of thermal expansion, CTE $=9.5 \mathrm{ppm} / \mathrm{K}[\underline{13}]$ and small refractive index change with $\mathrm{dn} / \mathrm{dt}=<1 \mathrm{ppm} / \mathrm{K}$ [8]. It is a reason why an increase of a resonant wavelength of a DBR element with temperature increase resulted mainly from thermal expansion of PTR glass, which increases the period of the volume grating. Small refractive index change cannot affect this process. A thermal shift of the Bragg wavelength of the PTR VBGs in the vicinity of $1 \mu \mathrm{m}$ is about $10 \mathrm{pm} / \mathrm{K}$. This shows that the local temperature increase in the focal area of the DBR element was about $35 \mathrm{~K}$. It is important to note that fine control of a DBR element with a TEC enables controlled emitting wavelength tuning within several hundreds of picometers. It was found that increase of pump power caused increase of divergence $\left(M^{2}\right)$ from 2 to 3.5. While no evidences of high-order transverse modes were found, this effect could be explained by thermal lensing resulting from uneven distribution of pumping power in the gain element.

PTR glass with $2 \%$ of $\mathrm{Yb}$ was used as an active element for an Yb-doped DFB laser. The resonant wavelength for the grating was chosen to be in the region of maximum gain $(\sim 1066 \mathrm{~nm})$, which was determined in external cavity lasing experiments with $\mathrm{Yb}$-doped PTR glasses. It was found that diffraction efficiency is close to $99.9 \%$ and the grating bandwidth is as narrow as $200 \mathrm{pm}$.

An Yb-doped PTR glass DFB laser does not use two discrete mirrors to form the optical cavity (as for conventional laser designs). The grating acts as the wavelength selective element and provides the feedback, reflecting light back into the cavity to form the resonator. The grating is constructed to reflect only a narrow band of wavelengths, and thus produce a single longitudinal lasing mode.

It was found that this Yb-doped PTR glass with such a VBG recorded in the whole volume shows lasing at a threshold of $4 \mathrm{~W}$ with no external feedback. CW laser emission was observed in both directions at the same wavelength. The results of the measurements of the power dependence of backward and forward signals are shown in Fig. 4(a). One can see that the maximum power in each direction reaches the value of $500 \mathrm{~mW}$ with slope efficiencies of $13 \%$ and $\sim 10 \%$ in the forward and backward directions, respectively. Optical-to-optical conversion efficiency was measured to be $\sim 3-3.5 \%$ for both directions. Saturation of the output power is related to the heating of the active element. Similar to the case of a Nd-doped PTR glass DBR laser, an increase of the pump power led to the change of the emitted wavelength [Fig. $\underline{4(\mathrm{~b})]}$ in the $\mathrm{Yb}$ doped DFB laser. A $150 \mathrm{pm}$ redshift was observed at absorber pump power of $8.5 \mathrm{~W}$. This value corresponds to heating of the DFB PTR laser of $15 \mathrm{~K}$.

An approximation of the free spectral range of the DFB laser is where the cavity length and physical length of the laser are equal $(15 \mathrm{~mm})$, given by $\Delta \lambda=\left(\lambda_{0}^{2} /(2 \times l \times n)\right)$. By using the known parameters of the cavity and glass $\left(\lambda_{0}=1066 \mathrm{~nm}, \quad n=1.5, \quad l=15 \mathrm{~mm}\right), \quad$ one can find
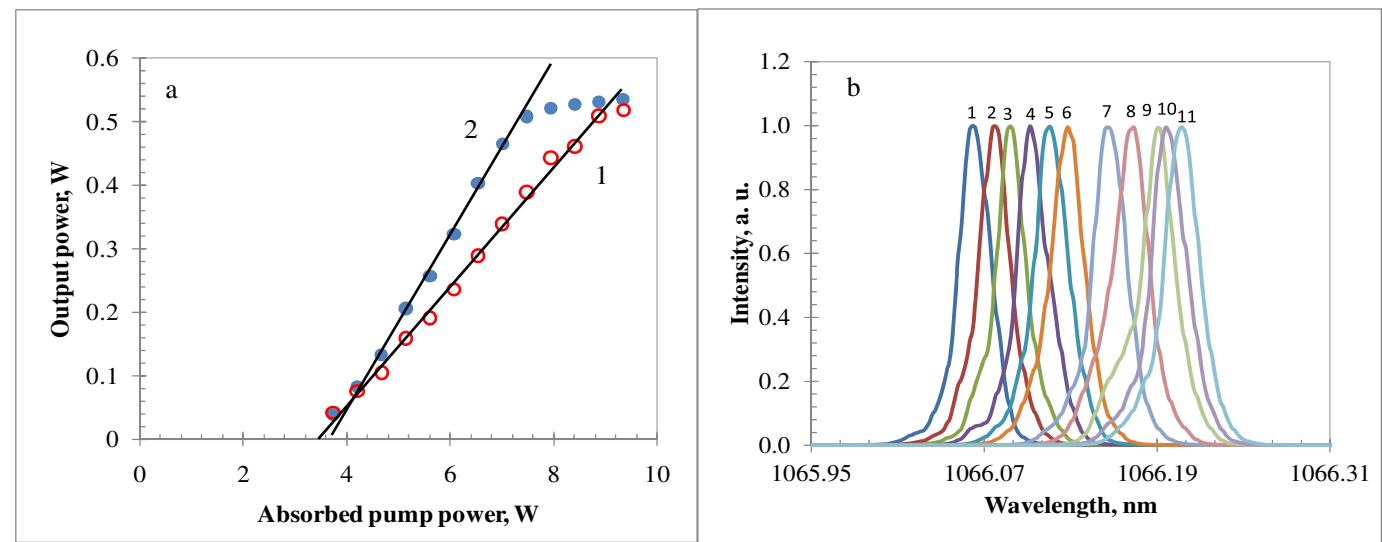

Fig. 4. Output parameters of Yb:DFB laser. (a) Output power versus absorbed pump power for backward (1) and forward (2) lasing. (b) Emission spectra at different levels of absorbed pump power: 1, 3.6 W; 2, 4.2 W; 3, 4.7 W; 4, 5.2 W; 5, 5.6 W; 6, 6 W; $7,6.5 \mathrm{~W} ; 8,7 \mathrm{~W} ; 9,7.5 \mathrm{~W} ; 10,8 \mathrm{~W} ; 11,8.5 \mathrm{~W}$. 
$\Delta \lambda=25 \mathrm{pm}$, while the spectral width of the DFB grating is about $100 \mathrm{pm}$. Using two different spectrum analyzers with resolution of 20 and 2 pm we observed a single emission line with spectral width equal to spectral resolution of the analyzers. To prove the laser operates in a single frequency regime, we used a scanning Fabry-Perot interferometer with a resolution of $7 \mathrm{MHz}$ and free spectral range of $1.5 \mathrm{GHz}(5.6 \mathrm{pm}$ at $1060 \mathrm{~nm})$. It was found that up to $5 \mathrm{~W}$ of absorbed pump power $(150-200 \mathrm{~mW}$ laser output) the laser operates in a single-frequency regime. The linewidth was measured to be $7.5 \mathrm{MHz}$, limited by the resolution of the scanning Fabry-Perot interferometer. The $M^{2}=2$ was measured in these conditions.

The pump power increase leads to the appearance of the second lasing line separated from the main line by $550-600 \mathrm{MHz}$ with corresponding degradation of $M^{2}$ up to 3.2 at maximum pump power.

A self-homodyne technique was used for linewidth characterization with higher resolution. In this case, a laser beam was split in two arms with both beams coupled to single-mode fibers. One of the fibers was connected with a $5 \mathrm{~km}$ delay line. Then, both arms were recombined by a fiber beam combiner. The beam combiner has two outputs with equal amounts from both arms in each output. One of the outputs is connected to the scanning Fabry-Perot interferometer to be sure that the laser operates in a single-frequency regime, and the second output is sent to a photodiode, which is connected to an electrical spectrum analyzer. Thus, the linewidth of the monolithic $\mathrm{Yb}$-DFB laser was measured to be $\leq 250 \mathrm{KHz}$.

Reflecting VBGs with diffraction efficiency exceeding 99\% were recorded directly in laser gain elements made from PTR glasses doped with $\mathrm{Nd}$ and $\mathrm{Yb}$. In the case of Nd-doped PTR glass, a DBR laser consisted of an external high reflecting conventional multilayer dielectric mirror and an output coupler within a gain element. $3 \mathrm{~W}$ peak power with a linewidth below $30 \mathrm{pm}$ (resolution of analyzer) and a slope efficiency of $15 \%$ was demonstrated. A monolithic solid-state DFB laser made in Yb-doped PTR glass with a VBG occupying the whole volume was demonstrated for the first time, to the best of our knowledge. Operating in a single longitudinal mode regime, it produces a linewidth $(<250 \mathrm{kHz})$ with a power of up to $150 \mathrm{~mW}$ in the forward and backward directions.

This work was partially supported by NASA and DARPA contracts NNX11CA87C and W31P4Q-12-C-0215.

\section{References}

1. H. Kogelnik and C. V. Shank, Appl. Phys. Lett. 18, 152 (1971).

2. C. V. Shank, J. E. Bjorkholm, and H. Kogelnik, Appl. Phys. Lett. 18, 395 (1971).

3. M. Nakamura, A. Yariv, H. W. Yen, S. Somekh, and H. L. Garvin, Appl. Phys. Lett. 23, 224 (1973).

4. R. M. Lammert, J. E. Ungar, S. W. Oj, H. Qi, J. S. Chen, and N. Bar Chaim, Electron. Lett. 34, 1663 (1998).

5. H. Wenzel, J. Fricke, A. Klehr, A. Knauer, and G. Erbert, IEEE Photon. Technol. Lett. 18, 737 (2006).

6. V. Ligeret, F.-J. Vermersch, S. Bansropun, M. Lecomte, M. Calligaro, O. Parillaud, and M. Krakowski, Proc. SPIE 6184, 61840A (2006).

7. O. M. Efimov, L. B. Glebov, L. N. Glebova, and V. I. Smirnov, "Process for production of high efficiency volume diffractive elements in photo-thermorefractive glass," U.S. patent 6,586,141 (July 1, 2003).

8. L. B. Glebov, J. Holography Speckle 5, 1 (2008).

9. L. Glebov, Rev. Laser Eng. 41, 684 (2013).

10. L. Glebova, J. Lumeau, and L. B. Glebov, Opt. Mater. 33, 1970 (2011).

11. Y. Sato, T. Taira, V. Smirnov, L. Glebova, and L. Glebov, Opt. Lett. 36, 2257 (2011).

12. O. M. Efimov, L. B. Glebov, and H. P. Andre, Appl. Opt. 41, 1864 (2002).

13. J. Deubener, H. Bornhöft, S. Reinsch, R. Müller, J. Lumeau, L. N. Glebova, and L. B. Glebov, J. Non-Cryst. Solids 355, 126 (2009). 